\title{
Micellization of Zonyl FSN-100 fluorosurfactant in aqueous solutions
}

\author{
Juraj Škvarla ${ }^{a}$, Mariusz Uchman ${ }^{a}$, Karel Procházka ${ }^{a}$, Zdeněk Tošner ${ }^{b}$, \\ Vasil M. Garamus ${ }^{\mathrm{c}}$, Stergios Pispas ${ }^{\mathrm{d}}$, Miroslav Štěpánek ${ }^{\mathrm{a}, *}$ \\ a Department of Physical and Macromolecular Chemistry, Charles University in Prague, Hlavova 2030, 12840 Prague 2, Czech Republic \\ b NMR Laboratory, Faculty of Science, Charles University in Prague, Hlavova 2030, 12840 Prague 2, Czech Republic \\ ${ }^{\mathrm{c}}$ Helmholtz-Zentrum Geesthacht, Centre for Materials and Coastal Research, Max-Planck Str. 1, D-21502 Geesthacht, Germany \\ ${ }^{\mathrm{d}}$ Theoretical and Physical Chemistry Institute, National Hellenic Research Foundation, 48 Vassileos Constantinou Avenue, 11635 Athens, Greece
}

\section{H I G H L I G H T S}

- Critical micelle concentration of FSN100 by surface tension measurement is $0.05 \mathrm{mmol} \mathrm{L}^{-1}$.

- FSN-100 forms core-shell spherical micelles with the radius of $3 \mathrm{~nm}$.

- FSN-100 micelles solubilize the fluorescent probe 1,6-diphenyl-1,3,5hexatriene.

\section{G R A P H I C A L A B S T R A C T}

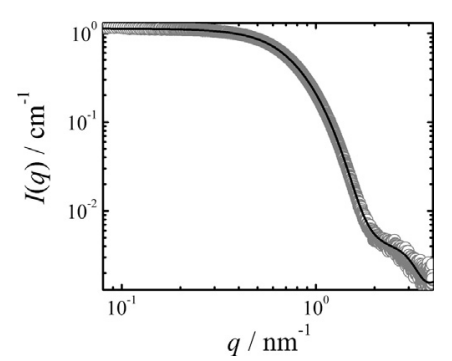

\begin{abstract}
A B S T R A C T
We report on micellization of nonionic fluorosurfactant Zonyl FSN-100 in aqueous solutions studied by means of NMR spectroscopy, light and small-angle X-ray scattering, surface tension measurements, isothermal titration calorimetry and fluorescence spectroscopy. The results allow for determination of basic parameters of Zonyl FSN-100 association like critical micellar concentration, size and association number of Zonyl FSN-100 micelles which have a core-shell structure with the core of fluorocarbon chains and the shell of oligo(ethylene oxide) chains. Time-resolved fluorescence spectroscopy studies using 1,6-diphenyl-1,3,5-hexatriene (DPH) as a fluorescent probe indicate that DPH can be solubilized in the micelles despite the general assumption about the immiscibility of hydrocarbon compounds with the fluorocarbon core of the micelles.
\end{abstract}

(c) 2013 Elsevier B.V. All rights reserved.

\section{Introduction}

Fluorinated amphiphilic compounds [1] have received great attention in the past several decades due to their properties which differ in many respects from their counterparts with hydrocarbon aliphatic chains and which are interesting for potential applications in medicine (oxygen delivery [2]) and technology (emulsifiers [3], hydrophobic surface layers [4]). Since the fluorocarbon chain is stiffer and bulkier than the hydrocarbon chain of the same length,

\footnotetext{
* Corresponding author. Tel.: +42022195 1290; fax: +420224919752.

E-mail address: stepanek@natur.cuni.cz (M. Štěpánek).
}

interfaces formed by fluorinated surfactants (FS) tend to be less curved than those of single-chain hydrocarbon surfactants, so that FS self-assemble in vesicles or wormlike micelles rather than in spherical micelles [5-7]. Low polarizibility of fluorine results in weaker dispersion forces between fluorocarbon tails as compared with hydrocarbons, which causes fluorocarbon chains being both hydrophobic and lipophobic [8]. Co-assembly of FS with hydrocarbon amphiphiles can lead to multicompartment structures in which fluorinated chains form segregated domains [9].

Zonyl FSN-100, $\mathrm{F}\left(\mathrm{CF}_{2} \mathrm{CF}_{2}\right)_{1-9} \mathrm{CH}_{2} \mathrm{CH}_{2} \mathrm{O}\left(\mathrm{CH}_{2} \mathrm{CH}_{2} \mathrm{O}\right)_{0-25} \mathrm{H}$ [10] is a representative of a nonionic fluorinated surfactant, which was produced by DuPont until 2010. Despite the fact that it was the subject of a number of studies dealing with its applications as an 
emulsification of plasmid DNA delivery systems [10], stabilization of gold nanoparticles [11] or formation of superhydrophobic selfassembled monolayers on gold [4], no study of its self-assembly in solution has been published so far.

In this article, we study association of Zonyl FSN-100 in aqueous solutions. We focus (i) on the process of formation of FSN100 micelles in water, which is followed by isothermal titration calorimetry and surface tension measurements and (ii) on the structure of FSN-100 micelles in water, which is characterized by means of light and small-angle X-ray scattering.

\section{Experimental}

\subsection{Materials}

Zonyl FSN-100 fluorosurfactant, 1,6-difenyl-1,3,5-hexatriene (DPH) and dioxane were purchased from Sigma-Aldrich. Deionized water was used for preparation of FSN-100 aqueous solutions. For fluorescence anisotropy measurements, $2 \mathrm{~mL}$ of $5 \mathrm{mM}$ stock solution of DPH in dioxane were added to $2 \mathrm{~mL}$ of $10 \mathrm{~g} \mathrm{~L}^{-1}$ Zonyl FSN-100 solution.

\subsection{NMR measurements}

${ }^{1} \mathrm{H},{ }^{13} \mathrm{C}$ and ${ }^{19} \mathrm{~F}$ NMR measurements were carried out at $25^{\circ} \mathrm{C}$ in $\mathrm{D}_{2} \mathrm{O}$ (Fluka, 99.5\% D) using a Bruker Avance III $600 \mathrm{MHz}$ and Varian Inova $400 \mathrm{MHz}$ instruments. For quantitative experiments (recycle delay 30 s), 2,2,2-trifluoroethanol (TFE) was used as an internal standard.

\subsection{Surface tension measurements}

Surface tension of FSN-100 solutions was measured at $25^{\circ} \mathrm{C}$ by the Wilhelmy plate method using a Krüss K100 tensiometer.

\subsection{Isothermal titration calorimetry measurements}

ITC measurements were performed at $25^{\circ} \mathrm{C}$ with a Nano ITC isothermal titration calorimeter (TA Instruments-Waters LLC, New Castle, DE). The microcalorimeter consisted of a reference cell and a sample cell both of $183 \mu \mathrm{L}$ volume ( 24 karat gold). The sample cell was connected to a $50 \mu \mathrm{L}$ syringe, the needle of which was equipped with a flattened, twisted paddle at the tip, which ensured continuous mixing of the solutions in the cell rotating at $250 \mathrm{rpm}$. Titration measurements were carried out by consecutive $2.06 \mu \mathrm{L}$ injections of an aqueous 2.1 mM Zonyl FSN-100 in aqueous solution from the syringe into the sample cell filled with water. A total of 24 consecutive injections were performed. The delay between two consecutive injections was $250 \mathrm{~s}$. These injections replace a part of the solution in the sample volume, and the changed concentration is considered in the calculation of the sample concentration. By this method, the differential heat of mixing is determined for discrete changes of composition. The data were analyzed using the NanoAnalyze software.

\subsection{Light scattering measurements}

The light scattering setup (ALV, Langen, Germany) consisted of a $22 \mathrm{~mW}$ He-Ne laser, operating at the wavelength $\lambda=632.8 \mathrm{~nm}$, an ALV CGS/8F goniometer, an ALV High QE APD detector and an ALV 5000/EPP multibit, multitau autocorrelator. Both static and dynamic LS measurements were carried out at $25^{\circ} \mathrm{C}$ in the angular range $30-150^{\circ}$ corresponding in aqueous solutions to the scattering vector magnitudes $q$ ranging from 6.8 to $25.6 \mu \mathrm{m}^{-1}$.

Refractive index increment measurements were carried out at $25^{\circ} \mathrm{C}$ using a Wyatt T-ReX differential refractometer. The aqueous solutions of FSN-100 in four different concentrations were pumped into the refractometer by a syringe driven by a 78-9100 C Cole Parmer syringe drive with a flow rate of $1 \mathrm{~mL} / \mathrm{min}$. The refractive index value was determined from the slope of the plot of the refractive index vs. concentration.

Dynamic light scattering (DLS) measurements were evaluated by fitting the measured electric field, $g^{(1)}(\tau, q)$, calculated from the measured time autocorrelation functions of the scattered light intensity, $g^{(2)}(\tau, q)$, as $g^{(1)}(\tau, q)=\left[g^{(2)}(\tau, q)-1\right]^{1 / 2}$.

\subsection{Small-angle X-ray scattering measurements}

SAXS experiments were performed on the P12 BioSAXS beamline at the storage ring PETRA III (EMBL/DESY, Hamburg, Germany) at $20^{\circ} \mathrm{C}$ using a Pilatus $2 \mathrm{M}$ detector and synchrotron radiation with the wavelength of $\lambda=0.1 \mathrm{~nm}$. The sample-detector distance was $3 \mathrm{~m}$, allowing for measurements in the $q$-range interval from 0.11 to $4.4 \mathrm{~nm}^{-1}$. The $q$ range was calibrated using the diffraction patterns of silver behenate. The experimental data were normalized to the incident beam intensity, corrected for non-homogeneous detector response, and the background scattering of the solvent was subtracted. The solvent scattering was measured before and after the sample scattering to control for possible sample holder contamination. Twenty consecutive frames (each $0.5 \mathrm{~s}$ ) comprising the measurement of the solvent, sample, and solvent were performed. From this background-corrected SAXS data, one-dimensional scattering curves were calculated. In order to verify that no artifacts as a result of radiation damage occurred, all scattering curves of a recorded dataset were compared to a reference measurement (typically the first exposure) and finally integrated by automated acquisition program [12] and analyzed by means of the SASfit 0.93 .2 software [13].

\subsection{Fluorescence spectroscopy measurements}

Fluorescence measurements were performed using a FluoroLog 3-22 spectrometer (Horiba - Jobin Yvon, France) equipped with a FluoroHub time-correlated single photon counting module and Glan-Thompson polarizers. The excitation source was a pulsed diode laser (NanoLED) with the emission maximum at $378 \mathrm{~nm}$ and the pulse fwhm about $200 \mathrm{ps}$, operated at the repetition frequency of $1 \mathrm{MHz}$. Obtained fluorescence decays were fitted by a double-exponential decay model using the Marquardt-Levenberg nonlinear least-squares method.

\section{Results and discussion}

\subsection{Determination of molar mass and chain lengths of FSN-100 by NMR}

Since the lengths of both hydrophilic and hydrophobic chains of FSN-100 are variable, we measured quantitative ${ }^{1} \mathrm{H},{ }^{13} \mathrm{C}$, and ${ }^{19} \mathrm{~F}$ NMR spectra of $20 \mathrm{mg} / \mathrm{mL} F S N-100$ solution in $\mathrm{D}_{2} \mathrm{O}$ with TFE as an internal standard in order to determine the fluorine and hydrogen content in the used FSN-100 sample; all the spectra are shown in the Supplementary material. The integral over the isolated signal of $\mathrm{CH}_{2} \mathrm{CF}_{2}$ protons was used to calculate the molar concentration of FSN-100 with respect to the known concentration of TFE. The measurements and calculations yield the molecular formula of the used FSN-100 sample, $\mathrm{CF}_{3}\left(\mathrm{CF}_{2}\right)_{\langle 7.4\rangle}\left(\mathrm{CH}_{2} \mathrm{CH}_{2} \mathrm{O}\right)_{\langle 13.7\rangle} \mathrm{H}$ corresponding to the average molar mass of $1044 \mathrm{~g} \mathrm{~mol}^{-1}$.

When studying solution self-assembly behavior of FSN-100, it is however, necessary to keep in mind that these values are averages and that the heterogeneity of both fluorocarbon and oligo(ethylene oxide) chains can lead not only to polydisperse micelles and less distinct unimer-to-micelles transitions but even to formation of 


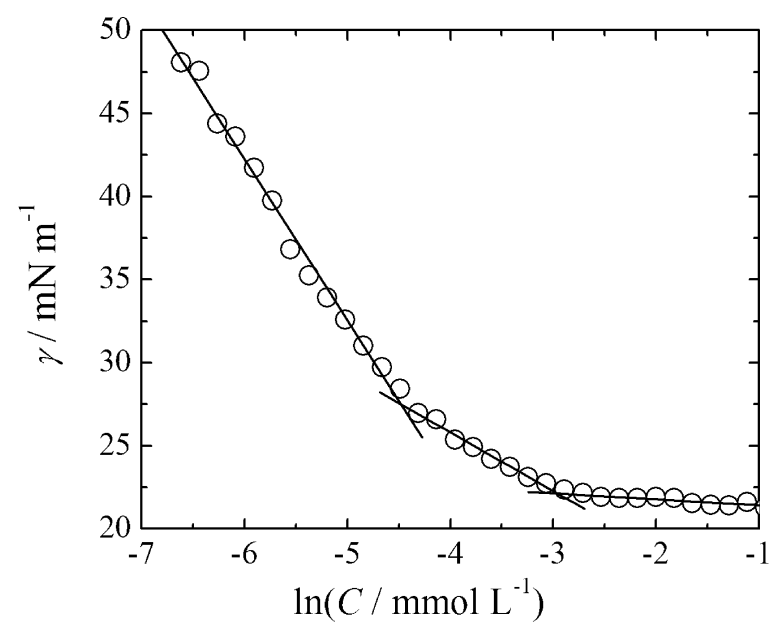

Fig. 1. Surface tension of FSN-100 aqueous solution, as a function of FSN-100 concentration.

self-assembled nanoparticles with various morphologies which will coexist in the solution at the same surfactant concentration.

\subsection{Measurements of critical micelle concentration}

In order to determine the critical micelle concentration of FSN100 , we employed surface tension, isothermal titration calorimetry and ${ }^{19} \mathrm{~F}$ NMR measurements. Fig. 1 shows the surface tension of FSN-100 aqueous solution, $\gamma$, as a function of the logarithm of the surfactant concentration up to $C=5 \mathrm{mg} / \mathrm{mL}$. The curve contains two breaks at 0.010 and $0.047 \mathrm{mmol} \mathrm{L}^{-1}$ which may indicate that the transition from unimers to micelles occurs gradually as a result of the presence of surfactants with various chain lengths in the solution. It is noteworthy that two breaks on the surface tension curve were also observed for Pluronic copolymers in aqueous solutions [14], where the presence of the low-concentration break was ascribed to the conformational transition of the polymer chain. It should be pointed out that in the case of FSN-100, the length of the chain is sufficient to exhibit a similar transition.

The slope of the $\gamma$ vs. $\ln C$ curve is proportional to the excess absorbed amount of the surfactant at air-water interface per unit area, $\Gamma=-(\partial \gamma / \partial \ln C)_{T, p} / R T$, where $R$ is the gas constant and $T$ temperature. Below the low-concentration break, the fit provides the value $\Gamma=3.91 \times 10^{-10} \mathrm{~mol} \mathrm{~cm}^{-2}$, corresponding to the surface area of $0.42 \mathrm{~nm}^{2}$. The high concentration break at $0.047 \mathrm{mmol} \mathrm{L}^{-1}$ indicates the cmc of the surfactant. Both parameters can be compared with those for the similar fluorosurfactant $\mathrm{CF}_{3}\left(\mathrm{CF}_{2}\right)_{5} \mathrm{CH}_{2} \mathrm{O}\left(\mathrm{CH}_{2} \mathrm{CH}_{2} \mathrm{O}\right)_{3} \mathrm{CH}_{3}$ with $\Gamma=4.37 \times 10^{-10} \mathrm{~mol} \mathrm{~cm}^{-2}$ and $\mathrm{cmc}=0.08 \mathrm{mmol} \mathrm{L}^{-1}[15]$.

The evaluation of ITC data (Fig. 2) is difficult because the demicellization contribution to the observed enthalpy changes decreases gradually with the FSN-100 concentration. While the inflection point indicating the $\mathrm{cmc}$ can be found, the plateau values are not accessible and the micellization enthalpy cannot thus be determined. This behavior can again be explained by the heterogeneity of FSN-100 which leads to complex multiple micellization equilibria, especially near the $\mathrm{cmc}$ region before the excess of uniform micelles is formed. The observed $\mathrm{cmc}$ value, $8.8 \times 10^{-2} \mathrm{mmol} \mathrm{L}^{-1}$, is roughly comparable with that from the tensiometric measurement.

As the third technique for the determination of $\mathrm{cmc}$, we used the method based on the fact that chemical shifts of NMR signals of the surfactant molecules in solution may differ significantly from those embedded in micelles. Due to the fast dynamic exchange of unimers among the micelles, the resulting chemical shift of such a

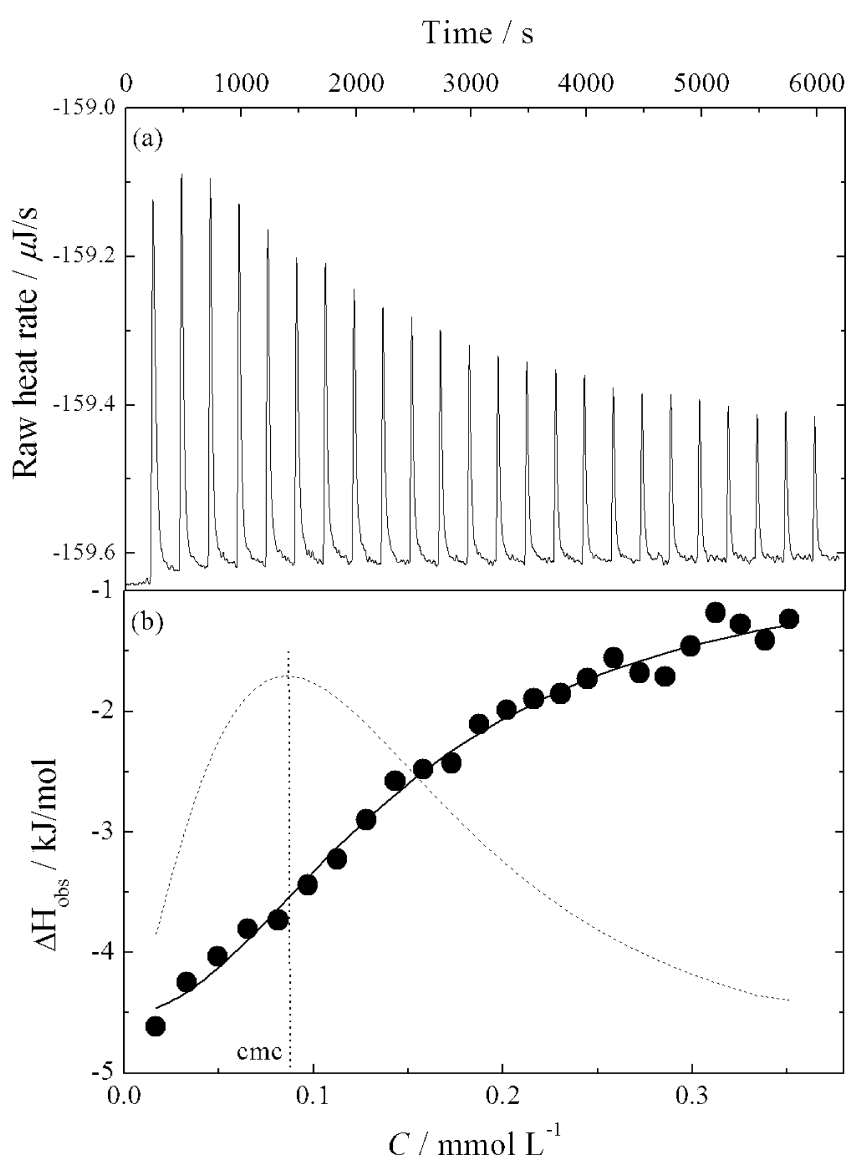

Fig. 2. (a) Heat flow rate during the injections of $2.06 \mathrm{~mL}$ portions of $2.1 \mathrm{mM}$ FSN100 solution into water. (b) Observed enthalpy change as a function of FSN-100 concentration (circles) and the fit by the sigmoidal function. The dashed curve is the first derivative of the fitted function.



Fig. 3. (a) ${ }^{19} \mathrm{~F}$ NMR spectra of $\mathrm{FSN}-100$ aqueous solution (region from -112 to $-116 \mathrm{ppm}$ ) in $\mathrm{D}_{2} \mathrm{O}$ at various concentrations (indicated above the curves, in $\mathrm{mmol} \mathrm{L}^{-1}$ ). (b) Concentration dependence of an environment-sensitive chemical shift of the signal shown in (a).

signal in a micellar system is the number average of the values for the micelle-bound surfactant and the free surfactant. Fig. 3a shows the concentration changes of the ${ }^{19} \mathrm{~F}$ NMR spectrum of FSN-100 in $\mathrm{D}_{2} \mathrm{O}$ in the region from -116 to $-112 \mathrm{ppm}$. One of the environmentsensitive $\mathrm{CF}_{2}$ signals appears at $-113.1 \mathrm{ppm}$ for the free FSN-100 in $0.05 \mathrm{mmol} \mathrm{L}^{-1}$ solution and at $-114.2 \mathrm{ppm}$ for FSN-100 in micelles in $5 \mathrm{mmol} \mathrm{L}^{-1}$. Above $0.1 \mathrm{mmol} \mathrm{L}^{-1}$, the signal moves upfield gradually with the increasing concentration as the micelles are formed 


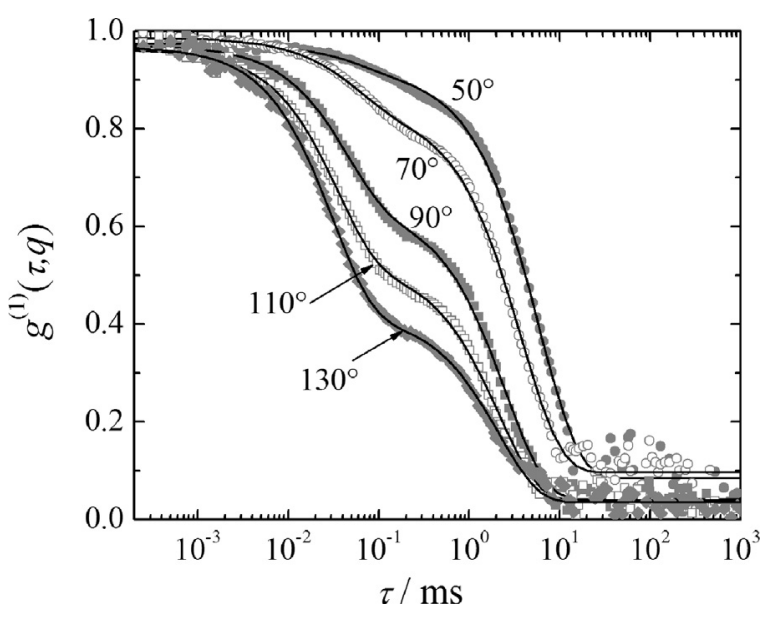

Fig. 4. Electric field autocorrelation functions for DLS of $20 \mathrm{~g} \mathrm{~L}^{-1} \mathrm{FSN}-100$ aqueous solution at various scattering angles.

(the concentration dependence is shown in Fig. 3b). Hence, the NMR measurement supports the results of surface tension and ITC measurements showing that the $\mathrm{cmc}$ of the system is close to $0.1 \mathrm{mmol} \mathrm{L}^{-1}$.

The second part of the study was aimed at the characterization of FSN-100 micelles in water at the room temperature. While it can be expected that FSN-100 self-assembly in aqueous solution has segregated fluorocarbon and oligo(ethylene oxide) chains, the shape of the particles is difficult to predict because of the polydispersity of the surfactant: The self-assembly of the stiff and bulky fluorocarbon chains requires a less curved interface (like in bilayered vesicles or cylindrical micelles), while the attached flexible hydrophilic chains with the relatively greater length favors spherical morphology. In order to elucidate the structure of FSN-100 self-assembly in water, we carried out LS and SAXS measurements.

\subsection{Light scattering}

Light scattering is less sensitive for studies of FS aqueous solution than for aqueous solutions of hydrocarbon surfactants because of low refractive index increments of FS in water (the refractive index increment of FSN-100 in water is only $0.080 \mathrm{~mL} / \mathrm{g}$ ) which results in low excess scattering intensities of FS aqueous solutions. In the case of FSN-100, reliable light scattering measurements in water can be done for concentrations greater than $\sim 1 \mathrm{mg} / \mathrm{mL}$ $\left(\sim 0.7 \mathrm{mmol} \mathrm{L}^{-1}\right.$, that is, above its $\left.\mathrm{cmc}\right)$, however, LS can still be used for determination of the hydrodynamic radius and molar mass of FSN-100 micelles.

Electric field autocorrelation functions, $g^{(1)}(\tau, q)$, of $20 \mathrm{~g} \mathrm{~L}^{-1} \mathrm{FSN}-$ 100 aqueous solution at various scattering angles are shown in Fig. 4. The $g^{(1)}(\tau, q)$ functions are distinctly double-exponential, with the relaxation times proportional to $q^{-2}$ indicating that both times correspond to fluctuations caused by diffusive motion of the scatterers. Fig. 5 shows the angular dependences of the apparent diffusion coefficients $D_{1}$ and $D_{2}$ and of the relative amplitude $f$ for the scattering of the particles with the diffusion coefficient $D_{1}$, obtained from fit of the $g^{(1)}(\tau, q)$ functions by the equation:

$g^{(1)}(\tau, q)=A\left[f e^{-D_{1} q^{2} \tau}+(1-f) e^{-D_{2} q^{2} \tau}\right]+B$

where $A$ is the amplitude and $B$ is the baseline constant. The average values of the diffusion coefficients in the angular range from $70^{\circ}$ to $130^{\circ}$ are $D_{1}=6.5 \times 10^{-11}$ and $D_{2}=1.04 \times 10^{-12} \mathrm{~m}^{2} \mathrm{~s}^{-1}$ which corresponds to hydrodynamic radii $R_{\mathrm{H}, 1}=3.1 \mathrm{~nm}$ and $R_{\mathrm{H}, 2}=194 \mathrm{~nm}$. Since the form factor for the large particles decreases strongly with increasing $q$, while the scattering from the small particles is

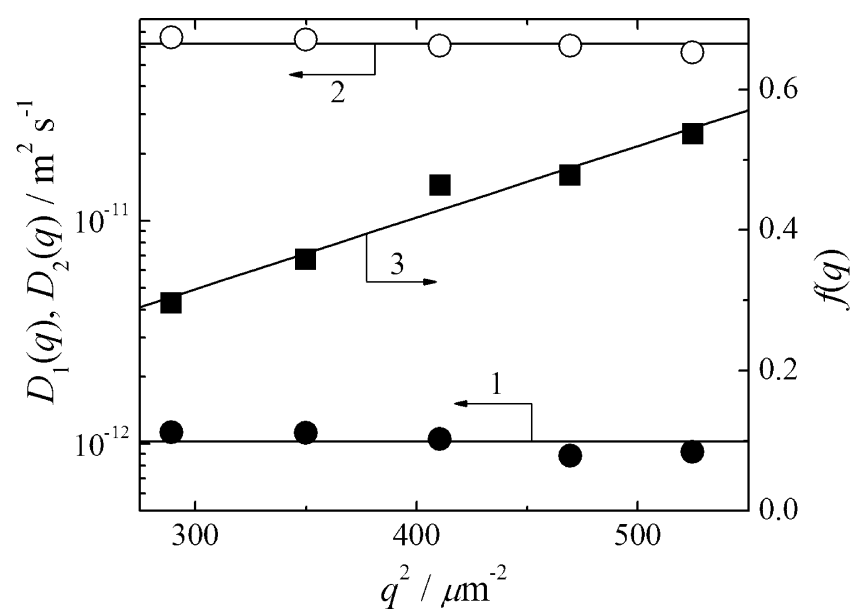

Fig. 5. Apparent diffusion coefficients $D_{1}, D_{2}$ (curves 1 and 2, respectively) and the relative amplitude of the mode with the diffusion coefficient $D_{1}$ (curve 3 ) as functions of $q^{2}$.

independent on the scattering angle, the relative amplitude $f$ increases with the increasing $q$.

The presence of two types of particles with very different sizes can be ascribed to the already mentioned heterogeneity of FSN-100 composition which may lead to the segregation of the surfactant molecules into different self-assemblies according to their hydrophobic and hydrophilic chain lengths. While the radius $R_{\mathrm{H}, 1}$ can be ascribed to FSN-100 micelles, the morphology of larger particles with the radius $R_{\mathrm{H}, 2}$ is unclear. We can hypothesize that these particles correspond to self-assemblies of FSN-100 molecules with shorter hydrophilic chains and longer hydrophobic chains (such as vesicles or wormlike micelles). However, as the difference in particles sizes reaches almost two orders of magnitude, the scattering cross-section of the micelles is negligible as compared with the large particles, which implies that at comparable scattering amplitudes of the two followed fluctuation processes, the amount of the large particles responsible for the slower fluctuation is negligible. For this reason we further focus on small FSN-100 micelles only.

In order to determine the molar mass of FSN-100 micelles, the scattering from the large particles has to be subtracted from the overall scattering from the sample. The contribution of the FSN100 micelles to the excess Rayleigh ratio, $\Delta R_{\theta}$, of the solution can be expressed by means of the relative amplitude $f$ as $\Delta R_{\theta}^{\text {mic }}=f \Delta R_{\theta}$. Taking into account that the mass fraction of FSN-100 in large particles is negligible, and that the form factor of the micelles in the $q$ range of the light scattering measurement is $P_{\text {mic }}(q) \approx 1$, the weight average molar mass, $M_{\mathrm{w}}$, of FSN-100 micelles can be found by fitting the data by the Zimm equation:

$\frac{K c}{\Delta R_{\theta}(c) f(c)}=\frac{1}{M_{\mathrm{w}}}+2 A_{2} c$

where $A_{2}$ is the second osmotic virial coefficient of the solution and $K=4 \pi^{2} n_{0}^{2}(\mathrm{~d} n / \mathrm{d} c)^{2} / \lambda^{4} N_{\mathrm{A}}$ is the optical constant in which $n_{0}$ is the refractive index of the solvent, $\mathrm{d} n / \mathrm{d} c$ is the refractive index increment of the solution and $N_{\mathrm{A}}$ is the Avogadro constant. Fig. 6 shows the values of $f$ and $K c / f \Delta R$ as functions of $c$ at the scattering angle of $90^{\circ}$. The fit of Eq. (2) to the data in Fig. 6 yields the value of the molar mass of the micelles, $M_{\mathrm{w}}=8.5 \times 10^{4} \mathrm{~g} \mathrm{~mol}^{-1}$, corresponding to the aggregation number, $N_{\text {agg }}=81$.

\subsection{Small-angle $X$-ray scattering}

SAXS measurement was used to confirm the assumption that FSN-100 forms spherical micelles composed of the compact core formed by $\mathrm{CF}_{3}\left(\mathrm{CF}_{2}\right)_{\langle 7.4\rangle}$ chains and the swollen shell of the hydrated 


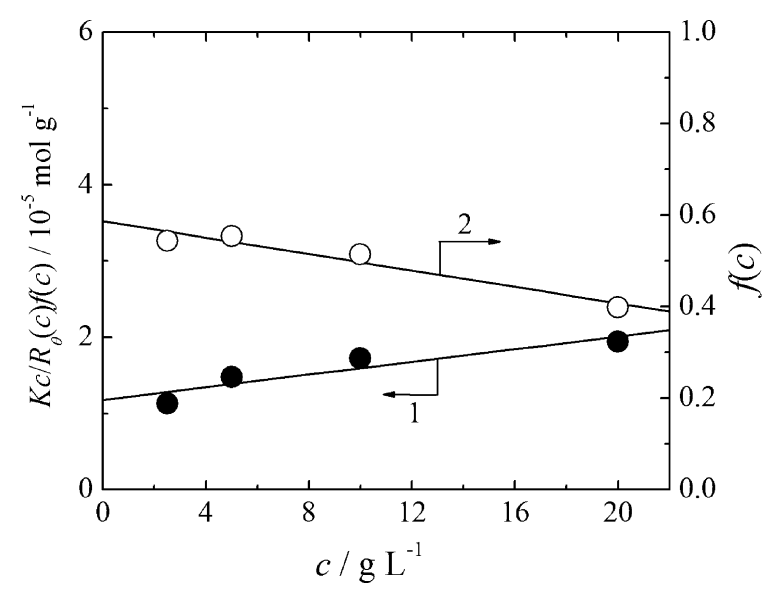

Fig. 6. Light scattering measurements of FSN-100 aqueous solutions at various FSN100 concentration, $c$ : reciprocal apparent molar mass from the SLS measurement, $K c / \Delta R_{\theta}(c) f(c)$ (curve 1 ), at the scattering angle $\theta=90^{\circ}$, and the relative amplitude $f(c)$ of the mode with the diffusion coefficient $D_{1}$ (curve 2 ).

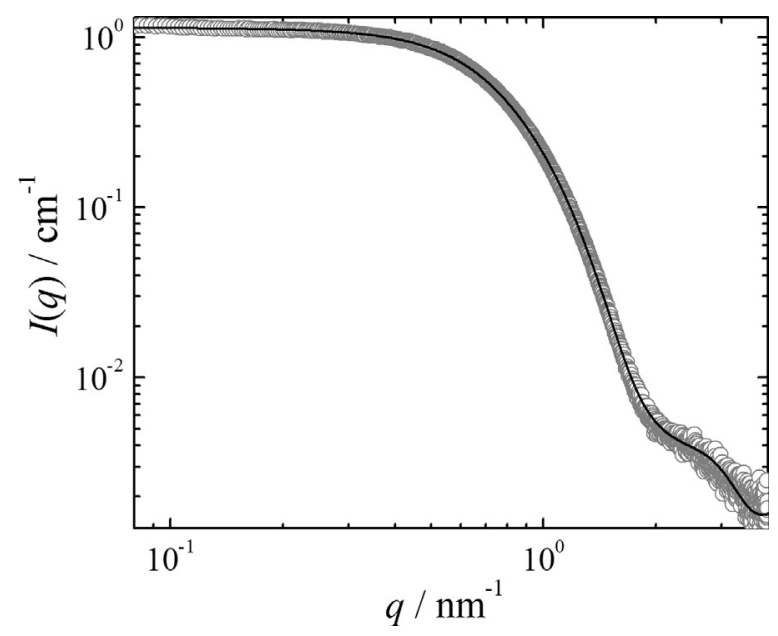

Fig. 7. SAXS curve of $10 \mathrm{mg} / \mathrm{mL}$ FSN-100 aqueous solutions. Open circles: experimental data, solid line: fit of the experimental data by the Pedersen-Gerstenberg model.

$\mathrm{H}\left(\mathrm{OCH}_{2} \mathrm{CH}_{2}\right)_{\langle 13.7\rangle}$ chains. The simplest model of the spherical core-shell particle, assuming both the homogenous core and the homogeneous shell, did not lead to satisfactory fits. Therefore, the scattering data were fitted by the Pedersen-Gerstenberg (PG) model [16] treating FSN-100 micelles as hard spheres with attached Gaussian chains. Since the influence of intermicellar interactions could not be neglected, the hard sphere structure factor [17] in the monodisperse approximation was added to the model. (The full expression for the scattering function is provided as Supplementary material for this article.) The scattering curve measured for $10 \mathrm{mg} / \mathrm{mL}$ FSN-100 aqueous solution and the fit of the experimental data by the PG model with the hard sphere structure factor are shown in Fig. 7. The parameters obtained from the fit are: (i) the core radius, $R=1.36 \mathrm{~nm}$, (ii) the gyration radius of a chain in the shell, $r=0.84 \mathrm{~nm}$, (iii) aggregation number, $N_{\mathrm{agg}}=102$, (iv) the excess scattering lengths of chains forming the core and the shell, $b_{\text {core }}=9.1 \times 10^{-12} \mathrm{~cm}$ and $b_{\text {shell }}=34.5 \times 10^{-12} \mathrm{~cm}$, the hard sphere interaction radius $R_{\mathrm{sph}}=4.32 \mathrm{~nm}$ and the excluded volume fraction of the micelles in the shell, $\varphi=2.56 \times 10^{-2}$.

Although the PG model was developed for block copolymer micelles with much longer chains than in the case of FSN-100, it appeared to be a good approximation of the radial dependence of the scattering length density in FSN-100 micelles.



Fig. 8. DPH emission decay (excitation: $378 \mathrm{~nm}$, emission, $425 \mathrm{~nm}$ ) in $10 \mathrm{mg} / \mathrm{mL}$ FSN-100 aqueous solution. Inset: DPH anisotropy decay in the same system.

Parameters obtained from the fit are in a good accordance with other experimental data for the FSN-100 solution, as we shown below.

(i) Gyration radius of the chains in the shell. The radius of the micelles, $R_{\text {mic }}=3.04 \mathrm{~nm}$, calculated as $R_{\mathrm{mic}}=R+2 r$, compares very well with the hydrodynamic radius, $R_{\mathrm{H}}=3.1 \mathrm{~nm}$, from DLS.

(ii) Aggregation number. Using the aggregation number, $N_{\mathrm{agg}}=102$, obtained from the fit and the mean molar mass of FSN-100, $M_{\mathrm{FSN}}=1044 \mathrm{~g} \mathrm{~mol}^{-1}$, we can calculate the molar mass of the micelles $M_{\text {mic }}$ SAXS $=10.7 \times 10^{5} \mathrm{~g} \mathrm{~mol}^{-1}$ which is roughly comparable with the value found by SLS, $M_{\text {mic }}=8.5 \times 10^{5} \mathrm{~g} \mathrm{~mol}^{-1}$.

(iii) Excluded volume fraction. Assuming the molar mass of $8.5 \times 10^{4} \mathrm{~g} \mathrm{~mol}^{-1}$ (SLS), the mass concentration of the solution, $c=10 \mathrm{mg} / \mathrm{mL}$, and the hard sphere interaction radius $R_{\mathrm{S}}=4.32 \mathrm{~nm}$, the excluded volume fraction, $\varphi^{\text {theor }}=$ $4 \pi R_{\mathrm{sph}}^{3} c N_{A} / 3 M_{\text {mic }}=2.39 \times 10^{-2}$ is similar to the values from the fit, $\varphi=2.56 \times 10^{-2}$.

As we pointed out in Section 1, fluorosurfactants tend to form vesicles or wormlike micelles rather than spherical micelles due to their bulky and rigid perfluorinated hydrophobic chains. However, the hydrophilic part of the FSN-100 molecule is a long chain whose molar volume exceeds that of the hydrophobic chain which leads to self-assembly with a highly curved interface. The SAXS data prove that the latter influence prevails and thus FSN-100 forms spherical core-shell micelles.

\subsection{Fluorescence measurements}

In order to study the interaction of FSN-100 micelles with low-molar-mass compounds, we carried out a series of fluorescence measurements using 1,6-diphenyl-1,3,5-hexatriene (DPH) as a hydrophobic fluorescent probe. Since DPH emission is environmentally sensitive due to solvent-induced energy shift effects on the close-lying excited states [18], DPH can be used as a probe for microenvironment polarity.

Fig. 8 shows fluorescence emission decay of $5 \mu \mathrm{M}$ DPH in $10 \mathrm{~g} \mathrm{~L}^{-1}$ aqueous solution of FSN-100 and its fit by the triple exponential model

$I(t)=\sum_{i=1}^{3} \alpha_{i} e^{-t / \tau_{i}}$ 
where $\alpha_{1}=0.152, \alpha_{2}=0.057$, and $\alpha_{3}=0.091$ are the amplitudes and $\tau_{1}=6.82 \mathrm{~ns}, \tau_{2}=2.52 \mathrm{~ns}$, and $\tau_{3}=0.60 \mathrm{~ns}$ are the lifetimes. The multiexponential decay behavior suggests that DPH is incorporated into FSN-100 micelles where it experiences different microenvironments due to different polarities and polarizibilities of the inner core, the interfacial layer and the shell. In order to corroborate this assumption, we performed the time-resolved fluorescence anisotropy measurement. Inset in Fig. 8 shows the anisotropy decay of $5 \mu \mathrm{M}$ DPH in $10 \mathrm{~g} \mathrm{~L}^{-1}$ aqueous solution of FSN-100 and its fit by the formula [19]:

$r(t)=\left(r_{0}-r_{\infty}\right)\left[\beta e^{-t / \varphi_{1}}+(1-\beta) e^{-t / \varphi_{2}}\right]+r_{\infty}$

where $r_{0}=0.388$ and $r_{\infty}=-0.005$ are the initial and residual anisotropies, $\varphi_{1}=4.32 \mathrm{~ns}$ and $\varphi_{2}=1.10 \mathrm{~ns}$ are the rotational correlation times and $\beta=0.47$ is the relative amplitude for the correlation time $\varphi_{1}$. The obtained $r_{0}$ value agrees with the earlier reported DPH anisotropy in hydrocarbon glasses at low temperatures [20], the residual anisotropy is zero within the range of the experimental error. The mean rotational correlation time, $\langle\varphi\rangle=\beta \varphi_{1}+(1-\beta) \varphi_{2}=2.64 \mathrm{~ns}$, can be used for the calculation of the volume of the rotating unit according to the formula $V=\langle\varphi\rangle k_{\mathrm{B}} T / \eta$, where $k_{\mathrm{B}}$ is the Boltzmann constant and $\eta$ is the solvent viscosity. The resulting value $V=1.09 \times 10^{-26} \mathrm{~m}^{3}$ corresponds to the volume of a sphere with the radius $2.95 \mathrm{~nm}$, which compares very well with the hydrodynamic radius of a FSN-100 micelle. This result proves that the DPH molecules are embedded in FSN micelles and their fluorescence depolarization dynamics is controlled by the rotational diffusion of the micelles. The fact that DPH is solubilized by FSN-100 is somehow surprising because hydrocarbon fluorophores such as pyrene were reported not to interact with FS micelles [21], so that the fluorescence studies of FS have been carried out using fluorophores with fluorocarbon chains [22].

\section{Conclusions}

We have studied aqueous solutions of the perfluorinated surfactant Zonyl FSN- 100 at $25^{\circ} \mathrm{C}$ by means of tensiometry, isothermal titration calorimetry, NMR spectroscopy, light and small-angle Xray scattering and by fluorescence spectroscopy. The study brought the following pieces of information about the association behavior of FSN-100 and about the structure of FSN-100 micelles:

(1) We have obtained the values of $\mathrm{cmc}$ of FSN-100 in aqueous solution by means of tensiometry, NMR and ITC, ranging from $0.05 \mathrm{mmol}^{-1}$ (tensiometry) to $0.1 \mathrm{mmol} \mathrm{L}^{-1}$ (NMR). The surface tension measurements have shown that adsorption of FSN-100 at the air-water interface is influenced by the conformation transition of the surfactant chains.

(2) We have studied the size and shape of FSN-100 micelles by a combination of static and dynamic LS and SAXS. Light scattering measurements have shown that trace amount of large particles (possibly vesicles) with the hydrodynamic radius about $200 \mathrm{~nm}$ coexist with FSN-100 spherical micelles with $R_{\mathrm{H}}=3 \mathrm{~nm}$. The SAXS of the micelles can be described by the Pedersen-Gerstenberg model, which suggests that their structure resembles that of block copolymer micelles. The fit allowed for determination of the core and shell dimensions. The aggregation number obtained from the PG model is in good accordance with the molar mass of FSN100 obtained from static light scattering. The formation of spherical micelles is caused by long hydrophilic chains of FSN-100 whose molar volumes exceed those of fluorocarbon ones.
(3) Time-resolved fluorescence measurements with DPH as a hydrophobic fluorescent probe have shown that DPH can be solubilized in FSN-100 micelles. This result is rather surprising because fluorocarbon domains in micelles formed by FS surfactants are both hydrophobic and lipophobic and it was reported that fluorescent probes do not solubilize in domains of fluorocarbon chains in FS micelles unless they possess a fluorocarbon tail.

\section{Acknowledgments}

The authors thank Dr. Edyta Kuliszewska (ICSO, KędzierzynKoźle, Poland) for surface tension measurements and Dr. Clement Blanchet (EMBL) for help during SAXS measurements. Financial support from the Ministry of Education of the Czech Republic (Long-Term Research Project MSM0021620857, Grant 7AMB13PL026) and Czech Science Foundation (Grants P106/12/0143 and P208/12/P236) is gratefully acknowledged.

\section{Appendix A. Supplementary data}

Supplementary data associated with this article can be found, in the online version, at http://dx.doi.org/10.1016/j.colsurfa.2013. 11.021.

\section{References}

[1] K. Matsuoka, Y. Moroi, Micellization of fluorinated amphiphiles, Curr. Opin. Colloid Interface Sci. 8 (2003) 227-235

[2] J.G. Riess, Blood substitutes and other potential biomedical applications of fluorinated colloids, J. Fluorine Chem. 114 (2002) 119-126.

[3] M.P. Krafft, Controlling phospholipid self-assembly and film properties using highly fluorinated components - fluorinated monolayers, vesicles, emulsions and microbubbles, Biochimie 94 (2012) 11-25.

[4] Y.G. Tang, J.W. Yan, F. Zhu, C.F. Sun, B.W. Mao, Comparative electrochemical scanning tunneling microscopy study of nonionic fluorosurfactant Zonyl FSN self-assembled monolayers on $\mathrm{Au}(111)$ and $\mathrm{Au}(100)$ : a potential-induced structural transition, Langmuir 27 (2011) 943-947.

[5] R. Oda, I. Huc, D. Danino, Y. Talmon, Aggregation properties and mixing behavior of hydrocarbon, fluorocarbon, and hybrid hydrocarbon-fluorocarbon cationic dimeric surfactants, Langmuir 16 (2000) 9759-9769.

[6] K. Wang, G. Karlsson, M. Almgren, T. Asakawa, Aggregation behavior of cationic fluorosurfactants in water and salt solutions. A cryo-TEM survey, J. Phys. Chem. B 103 (1999) 9237-9246.

[7] S. Rossi, G. Karlsson, S. Ristori, G. Martini, K. Edwards, Aggregate structures in a dilute aqueous dispersion of a fluorinated-hydrogenated surfactant system. A cryo-transmission electron microscopy study, Langmuir 17 (2001) 2340-2345.

[8] E.N.G. Marsh, Towards the nonstick egg: designing fluorous proteins, Chem. Biol. 7 (2000) R153-R157.

[9] Z.B. Li, E. Kesselman, Y. Talmon, M.A. Hillmyer, T.P. Lodge, Multicompartment micelles from ABC miktoarm stars in water, Science 306 (2004) 98-101.

[10] Z.G. Cui, W. Fountain, M. Clark, M. Jay, R.J. Mumper, Novel ethanol-influorocarbon microemulsions for topical genetic immunization, Pharm. Res. 20 (2003) 16-23

[11] Y.B. Zu, Z.Q. Gao, Facile and controllable loading of single-stranded DNA on gold nanoparticles, Anal. Chem. 81 (2009) 8523-8528.

[12] D. Franke, A.G. Kikhney, D.I. Svergun, Automated acquisition and analysis of small angle X-ray scattering data, Nucl. Instrum. Methods Phys. Res. A 689 (2012) 52-59.

[13] https://kur.web.psi.ch/sans1/SANSSoft/sasfit.html

[14] P. Alexandridis, V. Athanassiou, S. Fukuda, T.A. Hatton, Surface activity of poly(ethy1ene oxide)-block-poly(propy1ene oxide)-block-poly(ethy1ene oxide) copolymers, Langmuir 10 (1994) 2604-2612.

[15] J. Eastoe, A. Paul, R. Rankin, R. Wat, J. Penfold, J.R.P. Webster, Fluorinated nonionic surfactants bearing either $\mathrm{CF}_{3}-$, or $\mathrm{H}-\mathrm{CF}_{2}-$, terminal groups: adsorption at the surface of aqueous solutions, Langmuir 17 (2001) 7873-7878

[16] J.S. Pedersen, M.C. Gerstenberg, Scattering form factor of block copolymer micelles, Macromolecules 29 (1996) 1363-1365.

[17] J.K. Percus, G.J. Yevick, Analysis of classical statistical mechanics by means of collective coordinates, Phys. Rev. 110 (1958) 1-13.

[18] F. Schael, H.-G. Löhmannsröben, The deactivation of singlet excited all-trans1,6-diphenylhexa-1,3,5-triene by intermolecular charge transfer processes. 1 . Mechanisms of fluorescence quenching and of triplet and cation formation, Chem. Phys. 206 (1996) 193-210. 
[19] R. Esquembre, M.L. Ferrer, M.C. Gutiérrez, R. Mallavia, C.R. Mateo, Fluorescence study of the fluidity and cooperativity of the phase transitions of zwitterionic and anionic liposomes confined in sol-gel glasses, J. Phys. Chem. B 111 (2007) 3665-3673.

[20] C.R. Mateo, M.P. Lillo, J. Gonzalez-Rodriguez, A.U. Acuna, Molecular order and fluidity of the plasma membrane of human platelets from time-resolved fluorescence depolarization, Eur. Biophys. J. 20 (1991) 41-52.
[21] K. Stahler, J. Selb, P. Barthelemy, B. Pucci, F. Candau, Novel hydrocarbon and fluorocarbon polymerizable surfactants: synthesis, characterization and mixing behavior, Langmuir 14 (1998) 4765-4775.

[22] E. Szajdzinska-Pietek, M. Wolszczak, Time-resolved fluorescence quenching study of aqueous solutions of perfluorinated surfactants with the use of protiated luminophore and quencher, Langmuir 16 (2000) 1675-1680. 